Bull. Korean Math. Soc. 47 (2010), No. 6, pp. 1181-1188

DOI 10.4134/BKMS.2010.47.6.1181

\title{
THE $q$-ANALOGUE OF TWISTED LERCH TYPE EULER ZETA FUNCTIONS
}

\author{
LEECHAE JANG
}

\begin{abstract}
Volkenborn integrals ([8]) and fermionic invariant $q$-integrals ([12]) are introduced by T. Kim. By using these integrals, Euler $q$ zeta functions are introduced by T. Kim ([18]). Then, by using the Euler $q$-zeta functions, S.-H. Rim, S. J. Lee, E. J. Moon, and J. H. Jin ([25]) studied $q$-Genocchi zeta functions. And also Y. H. Kim, W. Kim, and C. S. Ryoo ([7]) investigated twisted $q$-zeta functions and their applications.

In this paper, we consider the $q$-analogue of twisted Lerch type Euler zeta functions defined by
\end{abstract}

$$
\zeta_{E, q, \varepsilon}(s)=[2]_{q} \sum_{n=0}^{\infty} \frac{(-1)^{n} \varepsilon^{n} q^{s n}}{[n]_{q}}
$$

where $0<q<1, \Re(s)>1, \varepsilon \in T_{p}$, which are compared with Euler $q$-zeta functions in the reference $([18])$. Furthermore, we give the $q$-extensions of the above twisted Lerch type Euler zeta functions at negative integers which interpolate twisted $q$-Euler polynomials.

\section{Introduction}

Let $p$ be a fixed odd prime number. Throughout this paper, $\mathbb{Z}_{p}, \mathbb{Q}_{p}$ and $\mathbb{C}_{p}$ will, respectively, the ring of $p$-adic rational integers, the field of $p$-adic rational numbers and the $p$-adic completion of the algebraic closure of $\mathbb{Q}_{p}$. The $p$-adic absolute value in $\mathbb{C}_{p}$ is normalized so that $|p|_{p}=\frac{1}{p}$. When one talks of $q$-extension, $q$ is variously considered as an indeterminate, a complex number $q \in \mathbb{C}$ or a $p$-adic number $q \in \mathbb{C}_{p}$. If $q \in \mathbb{C}$, one normally assumes $|q|<1$. If $q \in \mathbb{C}_{p}$, one normally assumes $|1-q|_{p}<p^{\frac{1}{1-p}}$ so that $q^{x}=\exp (x \log q)$ for each $x \in \mathbb{Z}_{p}$. We use the notation

$$
[x]_{q}=\frac{1-q^{x}}{1-q} \quad \text { and } \quad[x]_{-q}=\frac{1-(-q)^{x}}{1+q} \quad \text { cf. }[2-32]
$$

Received April 3, 2009; Revised February 13, 2010.

2000 Mathematics Subject Classification. 11B68, 11S80.

Key words and phrases. $p$-adic $q$-integral, $q$-Euler number and polynomials, $q$-Euler zeta functions, Lerch type $q$-Euler zeta functions.

This paper was supported by Konkuk University in 2010. 
for all $x \in \mathbb{Z}_{p}$. For a fixed odd positive integer $d$ with $(p, d)=1$, set

$$
\begin{aligned}
X & =X_{d}=\lim _{\overleftarrow{n}} \mathbb{Z} / d p^{n} \mathbb{Z}, X_{1}=\mathbb{Z}_{p}, \\
X^{*} & =\underset{\substack{0<a<d p \\
(a, p)=1}}{\rightarrow} \cup\left(a+d p \mathbb{Z}_{p}\right), \\
a+d p^{n} \mathbb{Z}_{p} & =\left\{x \in X \mid x \equiv a \quad\left(\bmod d p^{n}\right)\right\},
\end{aligned}
$$

where $a \in \mathbb{Z}$ lies in $0 \leq a<d p^{n}$. For any $n \in \mathbb{N}$,

$$
\mu_{q}\left(a+d p^{n} \mathbb{Z}_{p}\right)=\frac{q^{a}}{\left[d p^{n}\right]_{q}}
$$

is known to be a distribution on $\mathbb{Z}_{p}$, cf. [2-32].

We say that $f$ is a uniformly differentiable function at a point $a \in \mathbb{Z}_{p}$ and denote this property by $f \in U D\left(\mathbb{Z}_{p}\right)$, if the difference quotients

$$
F_{f}(x, y)=\frac{f(x)-f(y)}{x-y}
$$

have a limit $l=f^{\prime}(a)$ as $(x, y) \rightarrow(a, a)$, cf. [2-32].

The bosonic $p$-adic $q$-integral of a function $f \in U D\left(\mathbb{Z}_{p}\right)$ was defined by

$$
I_{q}(f)=\int_{\mathbb{Z}_{p}} f(x) d \mu_{q}(x)=\lim _{n \rightarrow \infty} \frac{1}{\left[p^{n}\right]_{q}} \sum_{x=0}^{p^{n}-1} f(x) q^{x} .
$$

In the sense of fermionic, let us define the fermionic $p$-adic $q$-integral as

$$
I_{-q}(f)=\int_{\mathbb{Z}_{p}} f(x) d \mu_{-q}(x)=\lim _{n \rightarrow \infty} \frac{1}{\left[p^{n}\right]_{-q}} \sum_{x=0}^{p^{n}-1} f(x)(-q)^{x},
$$

(see, $[2,7,11,12,18,25,28,32])$.

We note that $q$-Volkenborn integrals ([8]) and fermionic invariant $q$-integrals ([12]) are introduced by T. Kim. By using these integrals, Euler q-zeta functions are introduced by T. Kim ([18]). Then, by using the Euler $q$-zeta functions, S.-H. Rim, S. J. Lee, E. J. Moon, and J. H. Jin ([25]) studied $q$-Genocchi zeta functions. And also Y. H. Kim, W. Kim, and C. S. Ryoo ([7]) investigated twisted $q$-zeta functions and their applications.

In Section 2, we define the twisted $q$-Euler polynomials and numbers of higher order by using the fermionic $p$-adic $q$-integral. Let $\mathbb{N}$ be the set of positive integers. Let $T_{p}=\bigcup_{n \geq 1} C_{p^{n}}=\lim _{n \rightarrow \infty} C_{p^{n}}=C_{p^{\infty}}$ be the locally constant space, where $C_{p^{n}}=\left\{\varepsilon \mid \varepsilon^{p^{n}}=1\right\}$ is the cyclic group of order $p^{n}$. In Section 3, we consider the $q$-analogue of twisted Lerch type Euler zeta functions defined by

$$
\zeta_{E, q, \varepsilon}(s)=[2]_{q} \sum_{n=0}^{\infty} \frac{(-1)^{n} \varepsilon^{n} q^{s n}}{[n]_{q}},
$$

where $0<q<1, \Re(s)>1, \varepsilon \in T_{p}$, which are compared with Euler $q$-zeta functions in T. Kim ([18]). And we also give the $q$-extensions of the above 
twisted Lerch type Euler zeta functions at negative integers which interpolate twisted $q$-Euler polynomials.

\section{The twisted $q$-Euler numbers and polynomials}

For $m, k \in \mathbb{N}$ and $\varepsilon \in \mathbb{T}_{p}$, by using the fermionic $p$-adic $q$-integral, the twisted $q$-Euler polynomials $E_{m, q, \varepsilon}^{(-m, k)}$ of order $k$ in the variable $x$ in $\mathbb{C}_{p}$ are defined by

$$
\begin{aligned}
E_{m, q, w}^{(-m, k)}(x)= & \int_{\mathbb{Z}_{p}} \cdots \int_{\mathbb{Z}_{p}}\left[x+x_{1}+\cdots+x_{k}\right]_{q}^{m} \varepsilon^{x_{1}+\cdots+x_{k}} \\
& q^{-x_{1}(m+1)-\cdots-x_{k}(m+k)} d \mu_{-q}\left(x_{1}\right) \cdots d \mu_{-q}\left(x_{k}\right),
\end{aligned}
$$

(see $[4,5,20,26,27,28,31,29])$. From (2.1) with $x=0$, we can define the twisted $q$-Euler numbers of order $k$ as follows:

$$
E_{m, q, w}^{(-m, k)}=E_{m, q, w}^{(-m, k)}(0) .
$$

From (2.1), we can derive

$$
\begin{aligned}
E_{m, q, \varepsilon}^{(-m, k)}= & \lim _{n \rightarrow \infty} \frac{1}{\left[p^{n}\right]_{-q}^{k}} \sum_{x_{1}=0}^{p^{n}-1} \cdots \sum_{x_{k}=0}^{p^{n}-1}\left[x_{1}+\cdots+x_{k}\right]_{q}^{m} \\
& \times(-1)^{x_{1}+\cdots+x_{k}} \varepsilon^{x_{1}+\cdots+x_{k}} q^{-x_{1} m-\cdots-x_{k}(m+k-1)} \\
= & \frac{[2]_{q}^{k}}{(1-q)^{m}} \sum_{i=0}^{m}\left(\begin{array}{c}
m \\
i
\end{array}\right)(-1)^{i} \frac{1}{\left(1+\varepsilon q^{i-m}\right) \cdots\left(1+\varepsilon q^{i-m-k+1}\right)} \\
= & \frac{[2]_{q}^{k}}{(1-q)^{m}} \sum_{i=0}^{m}\left(\begin{array}{c}
m \\
i
\end{array}\right)(-1)^{i} \sum_{l=0}^{\infty}\left(\begin{array}{c}
k+l-1 \\
l
\end{array}\right)_{q}(-1)^{l} \varepsilon^{l} q^{(i-m-k+1) l} \\
= & {[2]_{q}^{k} \sum_{l=0}^{\infty}\left(\begin{array}{c}
k+l-1 \\
l
\end{array}\right)_{q}(-1)^{(1-m-k) l} \varepsilon^{l}(1-q)^{m} \sum_{i=0}^{m}\left(\begin{array}{c}
m \\
i
\end{array}\right)(-1)^{i} q^{i l} }
\end{aligned}
$$

Note that $(1-q)^{m} \sum_{i=0}^{m}\left(\begin{array}{c}m \\ i\end{array}\right)(-1)^{i} q^{i l}=\frac{\left(1-q^{l}\right)^{m}}{(1-q)^{m}}=[l]_{q}^{m}$. Thus, we obtain the following theorem.

Theorem 1. For $m, k \in \mathbb{N}$ and $\varepsilon \in T_{p}$, we have

$$
E_{m, q, \varepsilon}^{(-m, k)}=[2]_{q}^{k} \sum_{l=0}^{\infty}\left(\begin{array}{c}
k+l-1 \\
l
\end{array}\right)_{q}(-1)^{l} \varepsilon^{l} q^{(1-m-k) l}[l]_{q}^{m},
$$

where $\left(\begin{array}{c}z \\ w\end{array}\right)=\frac{z !}{w !(z-w) !}$ and $\left(\begin{array}{c}z \\ w\end{array}\right)_{q}=\frac{[z]_{q} !}{[w]_{q} ![z-w]_{q} !}$ for all $z, w \in \mathbb{N}$.

Note that $\lim _{q \rightarrow 1} E_{m, q, 1}^{(-m, k)}=E_{m}^{(k)}$ where the $E_{m}^{(k)}$ are ordinary Euler numbers of order $k$, which are defined by

$$
\left(\frac{2}{e^{t}+1}\right)^{k}=\sum_{n=0}^{\infty} E_{n}^{(k)} \frac{t^{n}}{n !}
$$


By (2.1) with $k=1$, it is easy to see that

$$
\begin{aligned}
E_{m, q, \varepsilon}^{(-m, 1)} & =\int_{\mathbb{Z}_{p}}\left[x+x_{1}\right]_{q}^{m} \varepsilon^{x_{1}} q^{-x_{1}(m+1)} d \mu_{-q}\left(x_{1}\right) \\
& =\int_{\mathbb{Z}_{p}}\left([x]_{q}+q^{x}\left[x_{1}\right]_{q}\right)^{m} \varepsilon^{x_{1}} q^{-x_{1}(m+1)} d \mu_{-q}\left(x_{1}\right) \\
& =\sum_{i=0}^{m}\left(\begin{array}{c}
m \\
i
\end{array}\right) q^{i x}[x]_{q}^{m-i} \int_{\mathbb{Z}_{p}}\left[x_{1}\right]_{q}^{i} \varepsilon^{x_{1}} q^{-x_{1}(m+1)} d \mu_{-q}\left(x_{1}\right) \\
& =\sum_{i=0}^{m}\left(\begin{array}{c}
m \\
i
\end{array}\right) q^{i x}[x]_{q}^{m-i} E_{i, q, \varepsilon}^{(-m, 1)} .
\end{aligned}
$$

Note that by (2.3) with $k=1$, we have

$$
E_{m, q, \varepsilon}^{(-m, 1)}=[2]_{q} \sum_{l=0}^{\infty}(-1)^{l} q^{-m l} \varepsilon^{l}[l]_{q}^{m} .
$$

Thus we obtain the following theorem.

Theorem 2. For $m, k \in \mathbb{N}$ and $\varepsilon \in T_{p}$, we have

$$
E_{m, q, \varepsilon}^{(-m, 1)}(x)=[2]_{q} \sum_{l=0}^{\infty} \sum_{i=0}^{m}\left(\begin{array}{c}
m \\
i
\end{array}\right)(-1)^{l} q^{-m l+i x} \varepsilon^{l}[x]_{q}^{m-i}[l]_{q}^{m} .
$$

\section{The twisted Lerch type Euler $q$-zeta functions}

In this section, we define twisted Lerch type Euler $q$-zeta function as follows:

$$
\zeta_{E, q, \varepsilon}(s)=[2]_{q} \sum_{n=0}^{\infty} \frac{(-1)^{n} \varepsilon^{n} q^{s n}}{[n]_{q}}
$$

where $0<q<1, s \in \mathbb{C}$ with $\Re(s)>1$, and $\varepsilon \in T_{p}$. Then we give the value $\zeta_{q, E, \varepsilon}(-m)$ for all $m \in \mathbb{N}$, which is an answer if the following problem: Are there twisted $q$-Euler polynomials which can be viewed as interpolating of $\zeta_{E, q, \varepsilon}(s)$ at negative integers and construct a new complex $q$-analogue of twisted Lerch type Euler zeta function.

From (2.1) with $k=1$, it is easy to see that

$$
\begin{aligned}
& E_{n, q, \varepsilon}^{(-n, 1)}=\int_{\mathbb{Z}_{p}}\left[x+x_{1}\right]_{q}^{m} \varepsilon^{x_{1}} q^{-x_{1}(m+1)} d \mu_{-q}\left(x_{1}\right) \\
& 2)=\frac{[2]_{q}}{[2]_{q^{d}}}[d]_{q}^{n} \sum_{i=0}^{d-1}(-1)^{i} \varepsilon^{i} q^{-n i} \int_{\mathbb{Z}_{p}} \varepsilon^{d t} q^{(m+1) d t}\left[\frac{x+i}{d}+t\right]_{q^{d}}^{n} \mu_{-q^{d}}(t),
\end{aligned}
$$

where $d, n$ are positive integers with $d \equiv 1(\bmod 2)$. Note that

$$
E_{n, q^{d}, \varepsilon^{d}}^{(-n, 1)}\left(\frac{x+i}{d}\right)=\int_{\mathbb{Z}_{p}} \varepsilon^{d t} q^{(m+1) d t}\left[\frac{x+i}{d}+t\right]_{q^{d}}^{n} \mu_{-q^{d}}(t) .
$$

Thus, by (3.2), we obtain the following property of twisted $q$-Euler polynomials: 
THE $q$-ANALOGUE OF TWISTED LERCH TYPE EULER ZETA FUNCTIONS 1185

Theorem 3. For $d, n \in \mathbb{N}$ with $d \equiv 1(\bmod 2)$ and $\varepsilon \in T_{p}$, we have

$$
E_{n, q, \varepsilon}^{(-n, 1)}(x)=\frac{[2]_{q}}{[2]_{q^{d}}}[d]_{q}^{n} \sum_{i=0}^{d-1}(-1)^{i} \varepsilon^{i} q^{-n i} E_{n, q^{d}, \varepsilon^{d}}^{(-n, 1)}\left(\frac{x+i}{d}\right)
$$

From (2.1) with $k=1$, we can derive

$$
\begin{aligned}
E_{m, q, w}^{(-m, 1)}(x) & =\int_{\mathbb{Z}_{p}} q^{-y(m+1)} \varepsilon^{y}[y+x]_{q}^{n} d \mu_{-q}(y) \\
& =\lim _{N \rightarrow \infty} \frac{1}{\left[p^{N}\right]_{-q}} \sum_{y=0}^{p^{N}-1} q^{-n y} \varepsilon^{y}[y+x]_{q}^{n}(-1)^{y} \\
& =\frac{1}{(1-q)^{n}} \sum_{l=0}^{n}\left(\begin{array}{c}
n \\
l
\end{array}\right)(-1)^{l} q^{x l} \lim _{N \rightarrow \infty} \frac{1+q}{1+q^{p^{N}}} \sum_{y=0}^{p^{N}-1}\left(q^{-n} \varepsilon q^{l}\right)^{y}(-1)^{y} \\
& =\frac{[2]_{q}}{(1-q)^{n}} \sum_{l=0}^{n}\left(\begin{array}{c}
n \\
l
\end{array}\right)(-1)^{l} q^{x l}\left(\frac{1}{1+q^{-n} \varepsilon q^{l}}\right) \\
& =\frac{[2]_{q}}{(1-q)^{n}} \sum_{l=0}^{n}\left(\begin{array}{c}
n \\
l
\end{array}\right)(-1)^{l} q^{x l} \sum_{m=0}^{\infty}(-1)^{m}\left(q^{-n} \varepsilon q^{l}\right)^{m} \\
& =[2]_{q} \sum_{m=0}^{\infty}(-1)^{m} q^{-n m} \varepsilon^{m} \frac{1}{(1-q)^{n}} \sum_{l=0}^{n}\left(\begin{array}{c}
n \\
l
\end{array}\right)(-1)^{l} q^{(m+x) l} .
\end{aligned}
$$

Note that

$$
[m+x]_{q}^{m}=\frac{1}{(1-q)^{n}} \sum_{l=0}^{n}\left(\begin{array}{l}
n \\
l
\end{array}\right)(-1)^{l} q^{(m+x) l}
$$

Thus we obtain the following another property of twisted $q$-Euler polynomials:

Theorem 4. For $n \in \mathbb{N}$ and $\varepsilon \in T_{p}$, we have

$$
E_{n, q, \varepsilon}^{(-n, 1)}(x)=[2]_{q} \sum_{m=0}^{\infty}(-1)^{m} q^{-n m} \varepsilon^{m}[m+x]_{q}^{n} .
$$

Let $F_{q, \varepsilon}(t, x)$ be the generating function of twisted $q$-Euler polynomials $E_{k, q, \varepsilon}^{(-k, 1)}(x)$ as follows:

$$
F_{q, \varepsilon}(t, x)=\sum_{k=0}^{\infty} E_{k, q, \varepsilon}^{(-n, 1)}(x) \frac{t^{k}}{k !}
$$


From (3.5) and (3.6), we can find the explicit series of the generating function $F_{q, \varepsilon}(t, x)$.

$$
\begin{aligned}
F_{q, \varepsilon}(t, x) & =\sum_{k=0}^{\infty} E_{k, q, \varepsilon}^{(-k, 1)}(x) \frac{t^{k}}{k !} \\
& =\sum_{k=0}^{\infty}\left([2]_{q} \sum_{m=0}^{\infty}(-1)^{m} q^{-k m} \varepsilon^{m}[m+x]_{q}^{k}\right) \frac{t^{k}}{k !} \\
& =[2]_{q} \sum_{m=0}^{\infty}(-1)^{m} \varepsilon^{m} \sum_{k=0}^{\infty} q^{-k m}[m+x]_{q}^{k} \frac{t^{k}}{k !} \\
& =[2]_{q} \sum_{m=0}^{\infty}(-1)^{m} \varepsilon^{m} e^{q^{-m}[m+x]_{q} t} .
\end{aligned}
$$

Finally, we assume that $q \in \mathbb{R}$ with $0<q<1$ and $\varepsilon \in T_{p}$. From (3.1) with $x=0$, we can see that the twisted Lerch type $q$-Euler zeta function is interpolating of twisted $q$-Euler numbers as follows:

Theorem 5. For $m \in \mathbb{N}$ and $\varepsilon \in T_{p}$, we have

$$
\zeta_{q, E, \varepsilon}(-m)=E_{m, q, \varepsilon}^{(-m, 1)} \text {. }
$$

From Theorem 4, we can define the $q$-extension of the twisted Lerch Euler zeta function as follows: For $s \in \mathbb{C}$ with $\Re(s)>1$ and $\varepsilon \in T_{p}$,

$$
\zeta_{q, E, \varepsilon}(s, x)=[2]_{q} \sum_{n=0}^{\infty} \frac{(-1)^{n} \varepsilon^{n} q^{s n}}{[n+x]_{q}^{s}} .
$$

Note that $\zeta_{q, E, \varepsilon}(s, x)$ is an analytic continuation in the whole complex plane. By (3.9) and Theorem 4, we can see that the $q$-extension of the twisted Lerch type Euler zeta function is interpolating of twisted $q$-Euler polynomials as follows:

Theorem 6. For $m \in \mathbb{N}$ and $\varepsilon \in T_{p}$, we have

$$
\zeta_{q, E, \varepsilon}(-m, x)=E_{m, q, \varepsilon}^{(-m, 1)}(x) .
$$

\section{References}

[1] L. C. Carlitz, q-Bernoulli numbers and polynomials, Duke Math. J. 15 (1948), 987-1000.

[2] M. Cenkci and M. Can, Some results on q-analogue of the Lerch zeta function, Adv. Stud. Contemp. Math. (Kyungshang) 12 (2006), no. 2, 213-223.

[3] M. Cenkci, Y. Simsek, and V. Kurt, Further remarks on multiple p-adic q-L-function of two variables, Adv. Stud. Contemp. Math. (Kyungshang) 14 (2007), no. 1, 49-68.

[4] L.-C. Jang, On a q-analogue of the p-adic generalized twisted L-functions and p-adic $q$-integrals, J. Korean Math. Soc. 44 (2007), no. 1, 1-10.

[5] _ Multiple twisted $q$-Euler numbers and polynomials associated with $p$-adic $q$ integrals, Adv. Difference Equ. 2008 (2008), Art. ID 738603, 11 pp.

[6] L. C. Jang, S. D. Kim, D. W. Park, and Y. S. Ro, A note on Euler number and polynomials, J. Inequal. Appl. 2006 (2006), Art. ID 34602, 5 pp. 
[7] Y.-H. Kim, W. Kim, and C. S. Ryoo, On the twisted q-Euler zeta function associated with twisted q-Euler numbers, Proc. Jangjeon Math. Soc. 12 (2009), no. 1, 93-100.

[8] T. Kim, On a q-analogue of the p-adic log gamma functions and related integrals, J. Number Theory 76 (1999), no. 2, 320-329.

[9] _ Some identities on the q-Euler polynomials of higher order and q-Stirling umbers by the fermionic p-adic integral on $\mathbb{Z}_{p}$, Russ. J. Math. Phys. 16 (2009), no. 4, 484-491.

[10] _ , q-Volkenborn integration, Russ. J. Math. Phys. 9 (2002), no. 3, 288-299.

[11] _ On Euler-Barnes multiple zeta functions, Russ. J. Math. Phys. 10 (2003), no. 3, 261-267.

[12] Analytic continuation of multiple q-zeta functions and their values at negative integers, Russ. J. Math. Phys. 11 (2004), no. 1, 71-76.

[13] _ Power series and asymptotic series associated with the q-analog of the twovariable p-adic L-function, Russ. J. Math. Phys. 12 (2005), no. 2, 186-196.

[14] _ Multiple p-adic L-function, Russ. J. Math. Phys. 13 (2006), no. 2, 151-157.

[15] _ q-generalized Euler numbers and polynomials, Russ. J. Math. Phys. 13 (2006), no. 3, 293-298.

[16] _ On the analogs of Euler numbers and polynomials associated with p-adic qintegral on $\mathbb{Z}_{p}$ at $q=-1$, J. Math. Anal. Appl. 331 (2007), no. 2, 779-792.

[17] $\_$-adic interpolating function for $q$-Euler numbers and its derivatives, J. Math. Anal. Appl. 339 (2008), no. 1, 598-608.

[18] _ Note on the Euler q-zeta functions, J. Number Theory 129 (2009), no. 7, 17981804.

[19] H. Ozden, I. N. Cangul, and Y. Simsek, Multivariate interpolation functions of higherorder q-Euler numbers and their applications, Abstr. Appl. Anal. 2008 (2008), Art. ID 390857, 16 pp.

[20] - Remarks on sum of products of $(h, q)$-twisted Euler polynomials and numbers, J. Inequal. Appl. 2008 (2008), Art. ID 816129, 8 pp.

[21] H. Ozden and Y. Simsek, A new extension of q-Euler numbers and polynomials related to their interpolation functions, Appl. Math. Lett. 21 (2008), no. 9, 934-939.

[22] H. Ozden, Y. Simsek, and I. N. Cangul, Euler polynomials associated with p-adic q-Euler measure, Gen. Math. 15 (2007), no. 2, 24-37.

[23] H. Ozden, Y. Simsek, S. H. Rim, and I. N. Cangul, A note on p-adic q-Euler measure, Adv. Stud. Contemp. Math. (Kyungshang) 14 (2007), no. 2, 233-239.

[24] S.-H. Rim and T. Kim, A note on p-adic Euler measure on $\mathbb{Z}_{p}$, Russ. J. Math. Phys. 13 (2006), no. 3, 358-361.

[25] S.-H. Rim, S. J. Lee, E. J. Moon, and J. H. Jin, On the q-Genocchi numbers and polynomials associated with q-zeta function, Proceedings of Jangjeon Math. Soc. 12 (2009), 261-268.

[26] Y. Simsek, $p$-adic twisted $q$-L-functions related to generalized twisted Bernoulli numbers, Russ. J. Math. Phys. 13 (2006), no. 3, 340-348.

[27] $ـ$, The behavior of the twisted $p$-adic $(h, q)$-L-functions at $s=0$, J. Korean Math. Soc. 44 (2007), no. 4, 915-929.

[28] _ On twisted $q$-Hurwitz zeta function and q-two-variable L-function, Appl. Math. Comput. 187 (2007), no. 1, 466-473.

[29] - Generating functions of the twisted Bernoulli numbers and polynomials associated with their interpolation functions, Adv. Stud. Contemp. Math. (Kyungshang) 16 (2008), no. 2, 251-278.

[30] Y. Simsek, V. Kurt, and D. Kim, New approach to the complete sum of products of the twisted $(h, q)$-Bernoulli numbers and polynomials, J. Nonlinear Math. Phys. 14 (2007), no. $1,44-56$. 
[31] Y. Simsek, O. Yurekli, and V. Kurt, On interpolation functions of the twisted generalized Frobenius-Euler numbers, Adv. Stud. Contemp. Math. (Kyungshang) 15 (2007), no. 2, $187-194$.

[32] H. M. Srivastava, T. Kim, and Y. Simsek, $q$-Bernoulli numbers and polynomials associated with multiple q-zeta functions and basic L-series, Russ. J. Math. Phys. 12 (2005), no. 2, 241-268.

Department of Mathematics and Computer Science

KonKuk University

Chunguu 380-701, Korea

E-mail address: leechae.jang@kku.ac.kr 